\title{
Use of triazolam and alprazolam as premedication for general anesthesia
}

\author{
Doyun Kim, Seongheon Lee, Taehee Pyeon, and Seongwook Jeong \\ Department of Anesthesiology and Pain Medicine, Chonnam National University Medical School, Gwangju, Korea
}

Background: Triazolam has similar pharmacological properties as other benzodiazepines and is generally used as a sedative to treat insomnia. Alprazolam represents a possible alternative to midazolam for the premedication of surgical patients. The purpose of this study was to evaluate the anxiolytic, sedative, and amnestic properties of triazolam and alprazolam as pre-anesthetic medications.

Methods: Sixty adult patients were randomly allocated to receive oral triazolam $0.25 \mathrm{mg}$ or alprazolam $0.5 \mathrm{mg}$ one hour prior to surgery. A structured assessment interview was performed in the operating room (OR), the recovery room, and the ward. The levels of anxiety and sedation were assessed on a 7-point scale $(0=$ relaxation to $6=$ very severe anxiety $)$ and a 5-point scale $(0=$ alert to $4=$ lack of responsiveness), respectively. The psychomotor performance was estimated using a digit symbol substitution test. As a memory test, we asked the patients the day after the surgery if they remembered being moved from the ward to the OR, and what object we had shown them in the OR.

Results: There were no significant differences between the groups with respect to anxiety and sedation. The postoperative interviews showed that $22.2 \%$ of the triazolam-treated patients experienced a loss of memory in the OR, against a $0 \%$ memory loss in the alprazolam-treated patients. In comparison with alprazolam $0.5 \mathrm{mg}$, triazolam $0.25 \mathrm{mg}$ produced a higher incidence of amnesia without causing respiratory depression.

Conclusions: Oral triazolam $0.25 \mathrm{mg}$ can be an effective preanesthetic medication for psychomotor performance.

Key Words: Alprazolam, Amnesia, Premedication, Triazolam.

\section{Introduction}

High levels of preoperative fear and anxiety correlate with various unfavorable outcomes, including increases in postopera-

Corresponding author: Seongwook Jeong, M.D., Ph.D

Department of Anesthesiology and Pain Medicine, Chonnam National University Medical School, 42, Jebong-ro, Dong-gu, Gwangju 501-757, Korea

Tel: 82-62-220-6895, Fax: 82-62-232-6294

E-mail: anesman@gmail.com

Received: January 16, 2015.

Revised: 1st, February 16, 2015; 2nd, March 5, 2015.

Accepted: March 10, 2015.

Korean J Anesthesiol 2015 August 68(4): 346-351

http://dx.doi.org/10.4097/kjae.2015.68.4.346 tive analgesic requirements, prolonged postanesthesia care unit or hospital stays, and delayed negative psychological effects [1]. In view of the high incidence and associated adverse outcomes in some patients groups [2], pharmacological (i.e., premedication) or psychological steps may be considered [1]. Benzodiazepines are extensively used as oral premedication as they present the advantage of avoiding painful intravenous or intramuscular injections. They differ in their ability to relieve primary or secondary (e.g., situational) anxiety, act as anticonvulsants, provide muscle relaxation, and induce sedation. As an anxiolytic drug, the benzodiazepine midazolam is the most commonly-used oral premedication [1]. However, alternatives are needed as the midazolam tablet is not available in South Korea anymore.

Ansseau et al. [3] built an anxiolytic index for oral benzodiazepines by using the ratio of the total primary and secondary anxiolytic activity scores divided by the total muscle relaxant

(c) This is an open-access article distributed under the terms of the Creative Commons Attribution Non-Commercial License (http://creativecommons.org/ licenses/by-nc/4.0/), which permits unrestricted non-commercial use, distribution, and reproduction in any medium, provided the original work is properly cited. 
and sedative activity scores. Using this index, one can select an appropriate anxioselective benzodiazepine. For example, prazepam scored the highest on the anxiolytic index. However, prazepam is inappropriate as a premedication as it has a delayed peak effect. Alprazolam at $0.5 \mathrm{mg}$ presents the second-highest anxioselective activity. In contrast with prazepam, it has an onset time of 1.4 hours and an elimination half-life of 10.6 hours in normal-weight subjects [4]. Given these pharmacokinetic properties and its major anxiety-reducing effects on patients with primary anxiety and panic attacks [5], alprazolam represents a possible alternative to midazolam for the premedication of surgical patients. After oral administration, it is rapidly absorbed and has a serum half-life of 12 to 15 hours for a single dose [6].

Triazolam is a popular premedication prescribed to highly anxious dental patients $[7,8]$ or patients with severe insomnia. Compared with other benzodiazepines, triazolam has a rapid onset, a short duration of action, and the ability to induce a somnolent state with minimal effects on the respiration and the myocardium $[9,10]$. It reaches peak blood level within approximately 1 hour, and its effects on anxiety, sedation, and amnesia are statistically significant against a placebo [3]. Despite its limited anxiolytic effects and psychologists' reluctance to prescribe it due to its induction of complex sleep-related behaviors in patients, triazolam's amnesic effect does not represent an adverse event for preanesthetic patients. Therefore, there is nothing intrinsically wrong with anesthesiologists selecting triazolam as a premedicant. A review of the clinical dental literature suggests that the oral or sublingual dose range for sedation is 0.25 to 0.5 $\mathrm{mg}$, and that it is effective when administered 30 to 45 minutes before the procedure [11].

This study was conducted with a prospective, randomized, and double-blinded design to compare the efficacy and adverse effects of triazolam and alprazolam as oral premedications.

\section{Materials and Methods}

\section{Subjects}

After obtaining approval from the Institutional Review Board and patients' written informed consent, sixty American Society of Anesthesiologist's physical status (ASA PS) 1 or 2 patients aged $20-55$ and weighing $55-80 \mathrm{~kg}$ who had been scheduled to receive elective surgery were recruited according to the protocol. Patients who had taken analgesic, sedative, antidepressant, or antiepileptic drugs within 1 week of the surgery were excluded. Other criteria for exclusion included drug allergies, chronic obstructive lung disease, pregnancy, and obesity (i.e., a body mass index [weight/height $\left.{ }^{2}\right] \geq 28$ ).

Before the beginning of the study, a sample size of 30 patients in each group was determined through a power analysis, based on the assumption that a difference of $30 \%$ in the two groups' amnesia would be clinically important. The 60 patients were randomly assigned to one of two groups: the triazolam group (Group T) received triazolam (0.25 mg Halcion ${ }^{\circledR}$; Pfizer, USA), and the alprazolam group (Group A) was premedicated with alprazolam (0.5 mg Xanax ${ }^{\circledR}$; Pfizer, USA) 60 to 90 minutes before the surgery. All the studied drugs were administered orally.

\section{Procedure}

In the operation room, the patients were monitored with electrocardiography, pulse oximetry, end-tidal carbon dioxide, noninvasive arterial pressure (Datex-Ohmeda $\mathrm{S} / 5^{\circledR}$, Planar Systems, Inc., Beaverton, OR, USA) and BIS (Aspect $2000^{\circledR}$, Aspect Medical Systems, Inc., Newton, MA, USA) monitors. Before the anesthesia, a venous catheter was inserted and an infusion of lactated Ringer's solution was administered at a rate of $10 \mathrm{ml} / \mathrm{kg} /$ h. Following full preoxygenation with $100 \%$ oxygen, anesthesia was induced with $2.0 \mathrm{mg} / \mathrm{kg}$ of intravenous propofol and a 2 $\mathrm{ng} / \mathrm{ml}$ remifentanil effect-site targeted infusion, followed by rocuronium $0.8 \mathrm{mg} / \mathrm{kg}$. When neuromuscular block was achieved, the trachea was intubated and the anesthesia was maintained with desflurane and a target-controlled infusion of remifentanil in order to prevent signs of inadequate anesthesia. If arterial hypotension developed, $5-10 \mathrm{mg} / \mathrm{kg}$ of ephedrine were administered intravenously. The drugs that were not mentioned in the protocol were excluded. The patients were actively warmed in order to keep their core temperature (esophageal) normothermic. In the postanesthesia care unit (PACU), the patients received standard postoperative care, including oxygen administration through a facial mask $(5 \mathrm{~L} / \mathrm{min})$. They also received appropriate pain, shivering, nausea, and vomiting control. The PACU discharge criteria included being awake and orientated, being able to breathe deeply and to cough freely, a blood pressure within $20 \%$ of the preoperative values, a temperature $\geq$ $36.0^{\circ} \mathrm{C}$, and the absence of shivering, minimal pain, and minimal nausea.

The effects of the studied drugs on the anxiety, sedation level, and psychomotor performance were assessed by an independent anesthesiologist or research nurse at four different time points: immediately after obtaining the patients' informed consent (baseline, Time 0), upon arrival in the operating room (Time A), just before discharge from the PACU (Time P), and before discharge from the hospital (Time D).

\section{Assessments and Measures}

Level of anxiety

The level of anxiety was assessed on a 7-point clinical global impression (CGI) scale [12] (0 = relaxation, 1 = apprehension, 
Psychomotor state (digit-symbol substitution test)

\begin{tabular}{|l|l|l|l|l|l|l|l|l|l|l|}
\hline 1 & 2 & 3 & 4 & 5 & 6 & 7 & 8 & 9 \\
\hline $\mathrm{V}$ & $\sqsupset$ & $\bullet$ & $\wedge$ & $\mathrm{X}$ & 7 & $\sqsubset$ & - & $\Gamma$ \\
\hline
\end{tabular}

\begin{tabular}{|l|l|l|l|l|l|l|l|l|l|l|l|l|l|l|l|l|l|l|l|}
\hline 2 & 1 & 3 & 1 & 2 & 1 & 3 & 1 & 4 & 2 & 4 & 2 & 5 & 1 & 4 & 3 & 5 & 2 & 6 & 2 \\
\hline & & & & & & & & & & & & & & & & & & & \\
\hline
\end{tabular}

$2=$ mild anxiety, $3=$ moderate anxiety, $4=$ manifest anxiety, 5 $=$ severe anxiety, $6=$ very severe anxiety). The degree of sedation was assessed on a 5 -point CGI scale $(0=$ alert, $1=$ arouses to voices, 2 = arouses with gentle tactile stimulation, $3=$ arouses with vigorous stimulation, $4=$ lack of responsiveness) and the Bispectral Index (in the OR only).

\section{Psychomotor performance}

The psychomotor performance was estimated with a Digit Symbol Substitution Test (DSST) [13]. The DSST is a subtest of the Wechsler Adult Intelligence Scale which is used to measure patients' general cognitive efficiency, working memory, and visual-motor coordination (Wechsler D. A manual for the Wechsler Adult Intelligence Scale. New York: Psychological Corporation, 1981). It takes the form of a timed pen-and-paper test in which patients are required to match numbers and symbols appropriately. The score is the time needed to match the 20 symbols correctly (Fig. 1).

\section{Memory}

As a memory test, we asked the patients one day after the surgery whether they remembered being moved from the ward to the OR, and what object (stethoscope, pen, or key chains) we had shown them in the OR. The test consisted in three questions which the patients had to answer with "yes" or "no". After the surgery, we asked the patients in the PACU and the ward about their satisfaction with the drug. The patients' satisfaction with the anxiety-reducing effect of the premedicant drug was assessed and scored as sufficient, insufficient, or indifferent ("don't know").

\section{Safety profiles}

The safety profiles of the two drugs were evaluated based on the incidence rate of adverse events, the vital signs, and a clinical laboratory test (complete blood cell count, urinalysis and alkaline phosphatase, aspartate aminotransferase, alanine aminotransferase, blood urea nitrogen, and creatinine levels, and coagulation parameters [prothrombin time, activated partial thromboplastin time, and platelet count]). All adverse events were assessed.
Table 1. Demographic Data

\begin{tabular}{lcc}
\hline \multicolumn{1}{c}{ Variable } & $\begin{array}{c}\text { Group T } \\
(\mathrm{n}=27)\end{array}$ & $\begin{array}{c}\text { Group A } \\
(\mathrm{n}=27)\end{array}$ \\
\hline Age $(\mathrm{yr})$ & $42.9 \pm 9.5$ & $42.7 \pm 10.2$ \\
Gender $(\mathrm{M} / \mathrm{F})$ & $54 \% / 46 \%$ & $45 \% / 55 \%$ \\
Weight $(\mathrm{kg})$ & $63.2 \pm 10.0$ & $65.4 \pm 8.0$ \\
Height $(\mathrm{cm})$ & $165 \pm 7.2$ & $164 \pm 7.0$ \\
\hline
\end{tabular}

Values are means \pm SD or proportions.

\section{Statistical analysis}

SPSS (Windows ver. 21.0, SPSS Inc., Chicago, IL) was used for the statistical analysis. Student t-test was used to compare normally distributed variables, and Mann-Whitney $U$ test was used to compare not-normally distributed continuous variables and ordinal variables. Categorical variables were compared with the $\chi^{2}$-test. Spearman correlation test was also used to assess the strength of association among continuous or ordinal variables. All the measured values were denoted as means \pm SD and numbers of patient. The statistical significance was defined as $\mathrm{P}<0.05$.

\section{Results}

Sixty patients were enrolled, and 6 patients dropped out in the course of the clinical trial period due to alterations of the operation schedule $(n=4)$ or personal refusal $(n=2)$; therefore, the data from a total of 54 patients were analyzed. The patients' demographic information is summarized in Table 1 . There were no differences between the groups in terms of age, sex, weight, or height.

There were no significant differences between the two groups in the time interval from administration of the medication to arrival in the operating room, and in the patients' bispectral index scores when they arrived in the operating room (Table 2).

There were no significant differences between the two groups with respect to the anxiety and sedation CGI scores (Table 2). The sedation CGI scores for time A (arrival in the operating room) and time P (before discharge from the PACU) did not differ significantly between the two groups (Table 2). 
Table 2. Perioperative Variables

\begin{tabular}{lcc}
\hline \multicolumn{1}{c}{ Variables } & $\begin{array}{c}\text { Group T } \\
(\mathrm{n}=27)\end{array}$ & $\begin{array}{c}\text { Group A } \\
(\mathrm{n}=27)\end{array}$ \\
\hline Time interval from medication to OR (min) $)$ & $55.0 \pm 29.1$ & $48.8 \pm 19.2$ \\
BIS in OR & $92.4 \pm 7.0$ & $92.8 \pm 6.5$ \\
Anxiety CGI score (0-6) & & \\
$\quad$ Time 0 & $0.8 \pm 0.5$ & $0.7 \pm 0.7$ \\
Time A & $0.8 \pm 0.6$ & $1.0 \pm 0.6$ \\
Time P & $0.2 \pm 0.4$ & $0.2 \pm 0.4$ \\
Time D & $0.0 \pm 0.1$ & $0.1 \pm 0.3$ \\
Sedation CGI scale & & \\
Time 0 & $0.0 \pm 0.1$ & $0.0 \pm 0.0$ \\
Time A & $0.1 \pm 0.3$ & $0.0 \pm 0.1$ \\
Time P & $0.1 \pm 0.3$ & $0.1 \pm 0.3$ \\
Time D & $0.0 \pm 0.1$ & $0.0 \pm 0.0$ \\
Psychomotor performance (sec) & & \\
Time 0 & $41.9 \pm 14.6$ & $47.5 \pm 21.9$ \\
Time A & $40.9 \pm 15.3 *$ & $41.2 \pm 17.2$ \\
Time P & $65.6 \pm 31.3 *$ & $51.0 \pm 20.2$ \\
Time D & $39.8 \pm 16.7 *$ & $36.1 \pm 14.2$ \\
Positive amnesia test (\%) & $22^{*}$ & 0 \\
Patient satisfaction score & $2.2 \pm 0.6^{*}$ & $1.7 \pm 0.3$ \\
\hline
\end{tabular}

Values are means \pm SD or proportions. CGI: clinical global impression scale, Time 0: immediately after obtaining informed consent (baseline), Time A: upon arrival in the operating room, Time P: just before discharge from the PACU, Time D: before discharge from the hospital. ${ }^{*} \mathrm{P}<0.05$ between groups.

The DSST completion time was significant in the triazolam group (group $\mathrm{T}$ ) when compared to that of the alprazolam group (group A) at all observed times (A, P and D) (Fig. 2).

The postoperative interviews showed that $22.2 \%(n=6)$ of the triazolam-treated patients experienced a loss of memory in the operating room, against a $0 \%$ memory loss in the alprazolam-treated patients $(\mathrm{P}=0.023)$. The patient satisfaction was significantly higher in the triazolam group $(\mathrm{P}=0.036)$, which was correlated with amnesia $(\mathrm{r}=0.34 ; \mathrm{P}=0.013)$. There were no cases of respiratory depression, delayed emergence, or other side effects in either group.

\section{Discussion}

In cases of minor surgery or patient groups with low risks of anxiety, anesthesiologists may not consider premedication with anxiety-reducing drugs. Therefore, most adults undergoing ambulatory or minor surgeries are not premedicated [14]. However, the majority of the patient population is willing to take a premedicant to reduce their anxiety. Eighty percent of the patients scheduled for a needle-guided breast biopsy expressed a preference for a combination of anxiety-reducing and hypnotic premedication before surgery [15].

Oral premedication with $0.5 \mathrm{mg}$ of alprazolam decreases anx-

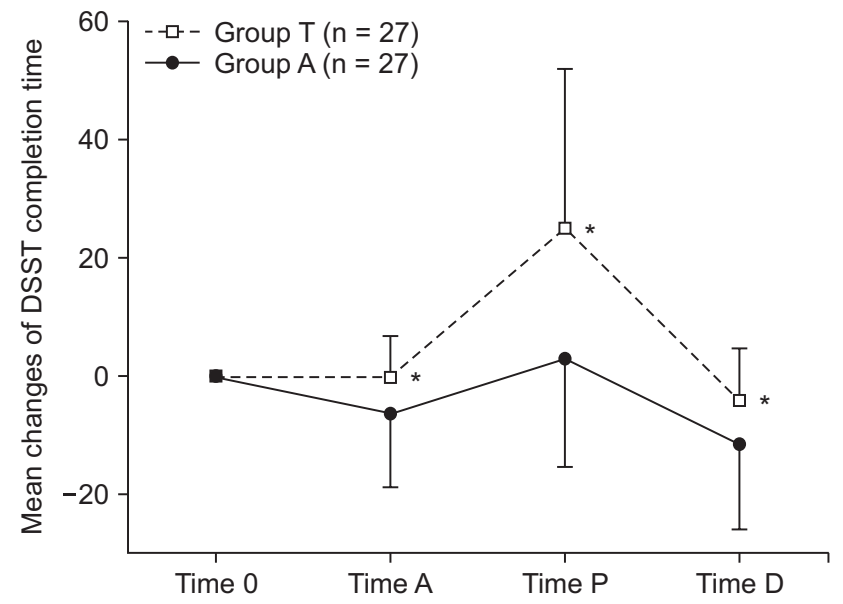

Fig. 2. Changes in the digit-symbol substitution test (DSST) completion time (mean, SD) relative to time 0 (baseline), as assessed at time A (arrival in the operating room), time $\mathrm{P}$ (discharge from the postanesthesia care unit) and time $\mathrm{D}$ (discharge from the hospital). $* \mathrm{P}<0.05$ as compared with Group A.

iety to the same extent as $7.5 \mathrm{mg}$ of oral midazolam. In $80 \%$ of patients, alprazolam was found to be as effective as midazolam for anxiety reduction, but not in its amnestic effects [4]. Choi et al. [16] reported that oral alprazolam attenuated the anxiety and preoperative stress responses of elective knee arthroscopic and reconstructive surgery patients under spinal anesthesia. They observed that the ACTH and cortisol levels in the operating room were significantly lower after alprazolam premedication before spinal anesthesia than in the placebo group.

By virtue of its fast onset of action and its short half-life, triazolam is usually used in the short-term treatment of acute insomnia, including jet lag. Despite its hypnotic, amnesic, anxiolytic, sedative, anticonvulsant, and muscle relaxant properties, most anesthesiologists do not favor triazolam as a premedication on account of its "hangover" effects. A meta-analysis demonstrated that the residual effects of the nighttime administration of triazolam, including sleepiness and impairment of the psychomotor and cognitive functions, may persist until the next day [17].

A few papers have provided reasonable evidence for the use of triazolam as appropriate anxiolytic premedication. Ehrich et al. [18] suggested that oral triazolam $(0.25 \mathrm{mg})$ was a safe and effective anxiolytic agent for endodontic patients. Yamakage et al. [19] reported that the administration of $0.25 \mathrm{mg}$ of oral triazolam was effective for smooth volatile anesthetic induction and comfort in adult patients. Recently, Iida et al. [20] demonstrated that triazolam triggered a high incidence of amnesia without causing respiratory depression.

The anxiolytic and amnesic actions of triazolam are sufficient to make it a viable alternative to midazolam. In this study, the oral administration of $0.25 \mathrm{mg}$ of triazolam was shown to 
be suitable as premedication for general anesthesia overall. It produced a higher incidence of amnesia than $0.5 \mathrm{mg}$ of alprazolam without causing respiratory depression. Milgrom et al. [7] reported that the oral administration of 0.375 or $0.50 \mathrm{mg}$ of triazolam was safe, and was highly effective in reducing both the anxious cognition and disruptive movements of highly-anxious dental patients, by affecting their episodic and implicit memory adversely. In terms of amnestic effects, the oral administration of $0.25 \mathrm{mg}$ of triazolam - i.e., a lower dose than in Milgrom et al. - was effective enough to induce a loss of memory in the operating room. Moreover, in the current study, the patient satisfaction was highly correlated with amnesia.

In comparison with the alprazolam group, the DSST completion time of the triazolam group at the time of arrival in the operating room did not improve despite the education effect. It was shown that triazolam had a stronger effect on patients' psychomotor functions than alprazolam. By contrast, the patients who had received alprazolam only demonstrated a subtle impairment in their psychomotor performance, and alprazolam did not induce amnesia in those patients. Even at a higher dose of $1.0 \mathrm{mg}$, no amnesic effect was noted for alprazolam [6].

The CGI (clinical global impression) scale for the assessment of sedation was not different between two groups, and most patients remained alert upon arrival in the operating room. This result shows that the sedative effects of triazolam $(0.25 \mathrm{mg})$ and alprazolam $(0.5 \mathrm{mg})$ are similar and minimal.

Other benzodiazepines available in oral preparations have been used for the premedication of adult outpatients. Preopera- tive discomfort and apprehension have been shown to be significantly reduced by diazepam $\left(0.25 \mathrm{mg} / \mathrm{kg}\right.$, Valium $\left.{ }^{\circledR}\right)[21,22]$. However, we did not select oral diazepam as an active comparator in this study, as its anxiolytic index score for $10 \mathrm{mg}$ is half that of alprazolam $0.5 \mathrm{mg}$ [3].

This study presented several limitations. First, we failed to enroll a placebo group of patients receiving no premedication. Second, we could not easily observe the sedated patients, as the statistical power was insufficient to detect the differences in sedation given that the calculation of the number of subjects focused on anxiolysis. Third, although there was no significant difference in the time from medication to arrival in the operating room (55 $\mathrm{min} / 48 \mathrm{~min}$ ), we could not control the medication time precisely. These limitations call for further studies.

In conclusion, triazolam produced a higher incidence of amnesia than alprazolam without causing respiratory depression. Upon discharge from the PACU, the triazolam group of patients experienced a significantly higher degree of satisfaction than the alprazolam group. The oral administration of $0.25 \mathrm{mg}$ of triazolam can be used effectively as an anesthetic premedication for the quality of anesthetic care.

\section{Acknowledgments}

We thank Inje Kim, Nana Choi and Suryeon Baek, clinical research coordinators of Anesthesiology research team of Chonnam National University Hospital.

\section{References}

1. Kain ZN, Mayes LC, Bell C, Weisman S, Hofstadter MB, Rimar S. Premedication in the United States: a status report. Anesth Analg 1997; 84: 427-32.

2. Kain ZN, Caldwell-Andrews AA, Maranets I, McClain B, Gaal D, Mayes LC, et al. Preoperative anxiety and emergence delirium and postoperative maladaptive behaviors. Anesth Analg 2004; 99: 1648-54.

3. Ansseau M, Doumont A, Diricq S. Methodology required to show clinical differences between benzodiazepines. Curr Med Res Opin 1984; 8 Suppl 4: 108-14.

4. De Witte JL, Alegret C, Sessler DI, Cammu G. Preoperative alprazolam reduces anxiety in ambulatory surgery patients: a comparison with oral midazolam. Anesth Analg 2002; 95: 1601-6.

5. Dawson GW, Jue SG, Brogden RN. Alprazolam: a review of its pharmacodynamic properties and efficacy in the treatment of anxiety and depression. Drugs 1984; 27: 132-47.

6. Franssen C, Hans P, Brichant JF, Noirot D, Lamy M. Comparison between alprazolam and hydroxyzine for oral premedication. Can J Anaesth 1993; 40: 13-7.

7. Milgrom P, Quarnstrom FC, Longley A, Libed E. The efficacy and memory effects of oral triazolam premedication in highly anxious dental patients. Anesth Prog 1994; 41: 70-6.

8. Jackson DL, Milgrom P, Heacox GA, Kharasch ED. Pharmacokinetics and clinical effects of multidose sublingual triazolam in healthy volunteers. J Clin Psychopharmacol 2006; 26: 4-8.

9. Bixler EO, Kales A, Manfredi RL, Vgontzas AN. Triazolam and memory loss. Lancet 1991; 338: 1391-2.

10. Weingartner HJ, Hommer D, Lister RG, Thompson K, Wolkowitz O. Selective effects of triazolam on memory. Psychopharmacology (Berl) 1992; 106: 341-5.

11. Jackson DL, Johnson BS. Inhalational and enteral conscious sedation for the adult dental patient. Dent Clin North Am 2002; 46: 781-802. 
12. Zaider TI, Heimberg RG, Fresco DM, Schneier FR, Liebowitz MR. Evaluation of the clinical global impression scale among individuals with social anxiety disorder. Psychol Med 2003; 33: 611-22.

13. Hindmarch I. Psychomotor function and psychoactive drugs. 1980. Br J Clin Pharmacol 2004; 58: S720-40.

14. Cammu G, Smith I. Day surgery, including the preoperative assessment of the patient: a UK experience by a Belgian anaesthetist. Acta Anaesthesiol Belg 2000; 51: 173-85.

15. van Vlymen JM, Sa Rego MM, White PF. Benzodiazepine premedication: can it improve outcome in patients undergoing breast biopsy procedures? Anesthesiology 1999; 90: 740-7.

16. Choi JC, Park SK, Kim SY, Yoon YS, Lee KH, Lee YB, et al. Oral Alprazolam Attenuates Preoperative Stress Responses to Regional Anesthesia. Korean J Anesthesiol 2007; 52: 132-7.

17. Vermeeren A. Residual effects of hypnotics: epidemiology and clinical implications. CNS Drugs 2004; 18: 297-328.

18. Ehrich DG, Lundgren JP, Dionne RA, Nicoll BK, Hutter JW. Comparison of triazolam, diazepam, and placebo as outpatient oral premedication for endodontic patients. J Endod 1997; 23: 181-4.

19. Yamakage M, Tsuchiya S, Ohtsuka N, Iwasaki S, Namiki A. Usefulness of oral hypnotic premedication for volatile induction of anesthesia in adults. J Anesth 2002; 16: 194-7.

20. Iida R, Kajiwara K, Kashiwai A, Kato J, Ogawa S. Comprehensive evaluation of the effect of triazolam on amnesia during the preoperative period. Masui 2011; 60: 67-74.

21. Jansen EC, Wachowiak-Andersen G, Munster-Swendsen J, Valentin N. Postural stability after oral premedication with diazepam. Anesthesiology 1985; 63: 557-9.

22. Jakobsen H, Hertz JB, Johansen JR, Hansen A, Kolliker K. Premedication before day surgery. A double-blind comparison of diazepam and placebo. Br J Anaesth 1985; 57: 300-5. 